\title{
Detection of High-Risk Human Papillomaviruses in the Prevention of Cervical Cancer in India
}

\author{
Krishnan Baskaran ${ }^{1 *}$, P Kranthi Kumar ${ }^{2}$, Santha Karunanithi ${ }^{1}$, Subramanian \\ Sethupathy ${ }^{1}$, B Thamaraiselvi ${ }^{3}$, S Swaruparani $^{3}$
}

\begin{abstract}
Human papillomaviruses (HPVs) are small, non-enveloped, double-stranded DNA viruses that infect epithelial tissues. Specific genotypes of human papillomavirus are the single most common etiological agents of cervical intraepithelial lesions and cervical cancer. Cervical cancer usually arises at squamous metaplastic epithelium of transformation zone (TZ) of the cervix featuring infection with one or more oncogenic or high-risk HPV (HRHPV) types. A hospital- based study in a rural set up was carried out to understand the association of HR-HPV with squamous intraepithelial lesions (SILs) and cervical cancer. In the present study, HR-HPV was detected in $65.7 \%$ of low-grade squamous intraepithelial lesions (LSILs), 84.6\% of high-grade squamous intraepithelial lesions (HSILs) and 94\% of cervical cancer as compared to $10.7 \%$ of controls. The association of HPV infection with SIL and cervical cancer was analyzed with Chi square test $(p<0.001)$. The significant association found confirmed that detection of HR-HPV is a suitable candidate for early identification of cervical precancerous lesions and in the prevention of cervical cancer in India.
\end{abstract}

Keywords: Human papillomavirus (HPV) - high-risk HPV - HPV testing - cervical cancer

Asian Pac J Cancer Prev, 16 (18), 8187-8190

\section{Introduction}

Human papillomaviruses (HPVs) are small, nonenveloped, double-stranded DNA viruses that infect epithelial tissues (Villa, 2006). HPV infection is one of the sexually transmitted diseases that occur through skin to skin contact (Fey and Beal, 2004; Bosch et al., 2006). More than 200 different HPV genotypes have been recognized (Burd, 2003) and one hundred eighteen have been characterized on the basis of L1 DNA sequence (Villiers et al., 2004). Out of these, around 40 of them are commonly found in genital tracts (Muñoz et al., 2003; Szostek et al., 2008). Various studies across the globe in the last two decades have consistently shown that specific genotypes of human papillomavirus are the single most etiological agents of cervical intraepithelial lesions (precancerous lesions) and cervical cancer. (Schiffman et al.,1993; Bosch et al., 2002). The cervical intraepithelial lesion is referred histologically as cervical intraepithelial neoplasia (CIN) or cytologically as squamous intraepithelial lesion (SIL) (Duarte-Franco and Franco, 2004). We adopted the cytological classification for defining cervical intraepithelial lesions.

Cervical cancer is the fourth most common cancer affecting women with an estimated 528, 000 new cases and an estimated 266,000 deaths worldwide in 2012 (GLOBOCAN 2012). Approximately $80 \%$ of all cervical cancers are squamous cell carcinoma (SCC) and 10 to $15 \%$ constitutes adenocarcinoma (ADC), around three present contributes to adenosquamous carcinoma (ADSC) and the remaining one to two percent forms neuroendocrine carcinoma ( $\mathrm{Lu}$ and Burk, 2000; Tjalma et al., 2005). Most of our study subjects were found to be SCC. Cervical cancer usually arises at squamous metaplastic epithelium of transformation zone (TZ) of the cervix with one or more of oncogenic or high-risk HPV (HR-HPV) types (Moscicki et al., 2006; Schiffman et al., 2007). The carcinogenesis tends to evolve slowly as HPV transmission, viral persistence, progression to precancerous lesion and invasion (Schiffman et al., 2007). So, the detection of HPV infection would identify a woman with a potential risk of cervical cancer and hence early intervention. The present study, therefore, was designed to identify the presence of HR-HPVs in SIL to cervical cancer and assess their clinical utility in the prevention of cervical cancer.

\section{Materials and Methods}

A total of 156 subjects within the age group of 30

${ }^{1}$ Department of Biochemistry, Rajah Muthiah Medical College \& Hospital, Annamalai University, Annamalainagar, ${ }^{3}$ Department of Gynecology and Obstetrics, Thanjavur Medical College, Thanjavur, Tamil Nadu, ${ }^{2}$ Department of Genetics, Narayana Medical College \& Hospital, Nellore, Andhra Pradesh, India*For correspondence: drjashmi@gmail.com 
to 65 years who were screened for the presence of HPV infection by polymerase chain reaction (PCR) in outpatient clinic in the Department of Gynecology and Obstetrics, Rajah Muthiah Medical College and Hospital, Annamalai University, Annamalainagar, Tamil Nadu, India and in the Department of Gynecology and Obstetrics, Thanjavur Medical College, Thanjavur, Tamil Nadu, India, from August 2008 to July 2010. The study subjects belonged to the rural population of Cuddalore and Thanjavur districts of Tamil Nadu, India. Subjects with history of hysterectomy / conization / previous treatment of cervical cancer, and currently undergoing any treatment for cervical diseases, presently pregnant, suffering from any serious and systemic illness like cardiac disease, any other malignancy and other sexually transmitted diseases were excluded. The study was approved by the Institutional Human Ethics Committee (IHEC) and the informed consent was obtained from each subject.

Cervical scrapings were collected using a sterile disposable cervical brush (Astra Scientific Systems Pvt. Ltd. Kerala, India) in a sample collection buffer [Phosphate buffer saline (PBS) $\mathrm{pH}$ 7.4] for the detection of HPV DNA. Biopsy specimens were obtained using a punch biopsy in neutral- buffered formalin [(NBF) (10\%)] for histopathological studies. The histopathological grading was performed according to WHO classification. There were a total of 67 subjects with cervical cancer. They consisted of 65 SCC and two ADSC. SIL was classified according to Bethesda System 2001 (Solomon et al., 2002). There were a total of 61 subjects with SIL: 26 subjects with high-grade squamous intraepithelial lesion (HSIL) while 35 with low-grade squamous intraepithelial lesion (LSIL). 28 subjects, who were posted for hysterectomy with non-cervical diseases such as uterine prolapse, were chosen as controls.

\section{Amplification of HPV DNA}

Detection of HPV infection with genotyping was performed by PCR (ApmliGenei HPV detection kit, Bangalore Genei, Bangalore, India). This kit detects eight HR-HPV types: HPV 16, 18, 31, 33, 35, 4552 and 58. These eight HR-HPV types in the descending order of frequency are: HPV 16, 18, 45, 31, 33, 52, 58, 35 and they are responsible for about $90 \%$ of all cervical cancers worldwide (Munõz et al., 2006). Genomic DNA from cervical scraps was extracted and HPV DNA was amplified in Eppendorf, Gradient Master Cycler (Germany) according to the manufacturer's instructions. The PCR product size varying from 230 to 270 base pairs (bps) was indicative of HPV infection. The PCR products were visualized and photographed (figure 1) in a gel documentation system (Bio-Rad, Gel Doc EQ, USA) and analyzed in quantity 1 software (Bio-Rad, USA). Genotyping was done based on the bps and later confirmed, if necessary, by automated DNA sequence analysis (Bangalore Genie, India).

\section{Statistical analysis}

The prevalence of HPV infection was expressed as percentage of HPV positives against all cases tested for HPV and the distribution of specific genotypes was also expressed as percentage. The association of HPV infection with SIL and cervical cancer was analyzed with Chi square test. In the present study, we have not analyzed the association of specific genotypes with SIL and cervical cancer. The statistical analysis of the data was performed in Systat version 12. The results were considered statistically significant if the $\mathrm{p}$ values were $\leq 0.05$.

\section{Results}

Table 1 shows the association of HPV infection with controls, LSIL, HSIL and cervical cancer. The association of HPV DNA was increased as the severity of cervical lesion increased and the association of HPV DNA was highest in cervical cancer. The HPV DNA was found in $10.7 \%$ of controls, $65.7 \%$ of LSIL, $84.6 \%$ of HSIL and $94 \%$ of cervical cancer. The HPV DNA was significantly associated with LSIL, HSIL and cervical cancer. Chi square value is highly significant $(p<0.001)$. The distribution of HPV genotypes among controls showed HPV 16 was the predominant type in $7.1 \%$ followed by HPV 18 detected in $3.6 \%$. Table 2 shows the distribution of specific genotypes in LSIL and HSIL. HPV 16 was the predominant type found in $34.3 \%$ followed by HPV 18 in

Table 1. Detection of HPV Infection among Study Subjects

\begin{tabular}{lcl}
\hline Study subjects & HPV positive \\
\hline Controls & $10.7(3)$ \\
LSIL & $65.7(23)$ \\
HSIL & $84.6(22)$ \\
Cervical cancer & 94 & $(63)$ \\
\hline
\end{tabular}

Data are expressed as percentage. Controls $(n=28)$; $\operatorname{LSIL}(n=35)$; HSIL ( $n=26) ; C C$ ( $n=67)$

Table 2. Distribution of HPV Types among LSIL and HSIL

\begin{tabular}{lrr}
\hline HPV types & \multicolumn{1}{c}{ LSIL } & HSIL \\
\hline 16 & $34.3(12)$ & $53.9(14)$ \\
18 & $11.4(4)$ & $15.4(4)$ \\
31 & $5.7(2)$ & $0(0)$ \\
33 & $0(0)$ & $0(0)$ \\
35 & $0(0)$ & $0(0)$ \\
45 & $0(0)$ & $3.8(1)$ \\
52 & $5.7(2)$ & $3.8(1)$ \\
58 & $8.6(3)$ & $7.7(2)$ \\
\hline
\end{tabular}

Data are expressed as percentage. LSIL ( $\mathrm{n}=35)$; HSIL $(\mathrm{n}=26)$

Table 3. Distribution of HPV Types among Cervical Cancer Cases

\begin{tabular}{lc}
\hline HPV types & Cervical cancer \\
\hline 16 & $59.7(40)$ \\
18 & $17.9(12)$ \\
31 & $2.9(2)$ \\
33 & $1.5(1)$ \\
35 & $1.5(1)$ \\
45 & $4.5(3)$ \\
52 & $1.5(1)$ \\
58 & $4.5(3)$ \\
\hline
\end{tabular}

Data are expressed as percentage. $C C(n=67)(S C C n=65 ; A D S C n=2)$ 


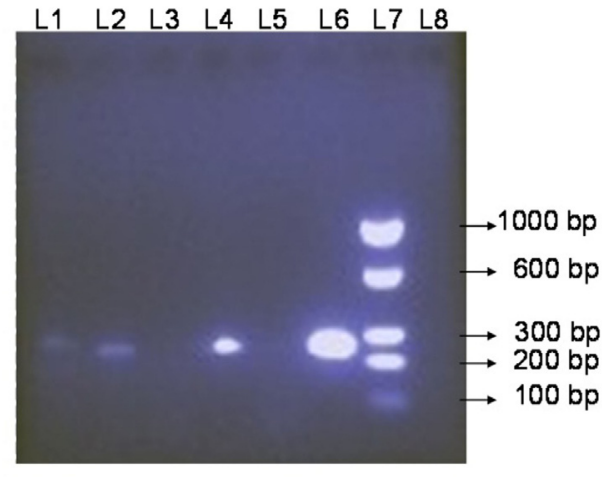

Lanes: L1) HPV18, L2) HPV16, L3) HPV Negative sample, L4) HPV16, L5) HPV Negative sample, L6) Positive control, L7) Molecular marker L8) Negative control

Figure 1. Agarose Gel Electrophoretogram Showing the HPV DNA by PCR

$11.4 \%$ of LSIL. The next predominant types were HPV 58 found in $8.6 \%$ followed by HPV 31 in $5.7 \%$ and HPV 52 in $5.7 \%$. HPV types 33,35 and 45 were not detected. HPV 16 was the major genotype found in $53.9 \%$ and HPV 18 in $15.4 \%$ of HSIL. The other genotypes detected were HPV 58 in $7.7 \%$, HPV 45 in $3.8 \%$ and HPV 52 in $3.8 \%$. Genotypes 31, 33 and 35 were not detected. Table 3 shows the distribution of specific genotypes in cervical cancer [consisted of 65 SCC and 2 ADSC; out of 2 ADSC 1 was HPV positive (HPV 18)]. HPV 16 was the major genotype found in $59.7 \%$ of cervical cancer followed by HPV 18 in $17.9 \%$, HPV 58 in $4.5 \%$, HPV 45 in $4.5 \%$, HPV 31 in $2.9 \%$, HPV 33 in $1.5 \%$, HPV 35 in $1.5 \%$ and HPV 52 in $1.5 \%$. No multiple infections were found in any of the control, LSIL, HSIL and cervical cancer.

\section{Discussion}

HPV has been proposed as the first ever identified necessary cause of a human cancer (Bosch et al., 2002) and it is the major causal agent of cervical cancer which unlikely develops in the absence of persistent HPV infection (Bosch et al., 2002). In other words, persistent infection with certain HR-HPV or oncogenic HPV is essential for the development of cervical intraepithelial lesions and cervical cancer. So, the detection of HR-HPV DNA in a clinical specimen will play a major role in screening, triage and follow up of cervical precancerous lesions (Heymans et al., 2011; Schiffman et al., 2011; Rijkaart et al., 2012; Dijkstra et al., 2014) In the present study, HPV DNA was detected in $65.7 \%$ of LSIL, $84.6 \%$ of HSIL, and $94 \%$ of cervical cancer as compared to $10.7 \%$ of controls. HPV DNA positivity increased as the lesion grade increased. HPV 16 and HPV 18 were the major genotypes prevalent in our study subjects as found in other parts of the world (Clifford et al., 2006; Smith et al., 2007; Bhatla et al., 2008; Bosch et al., 2008). Our results are in line with Guo et al. (2007) who demonstrated eight HR-HPV genotyping using quantitative real-time PCR. Significant association of HR-HPV infection suggests that detection of HPV might be useful in cervical cancer screening and in the management of cervical lesions.

Women infected with HR-HPV types are considered to be at higher risk for development of cervical cancer than those who are not infected with HR-HPV or infected with low-risk HPV types (Szosteket al., 2008). Therefore, detection of HPV infection may facilitate early identification of women at high risk of developing cervical cancer. Papanicolaou smear (Pap smear) has been the cervical screening test for decades. However, it has the limitation of lower sensitivity (Wright, 2007) and also cervical cytology based program requires tremendous training, infrastructure, and repeated visit by the women for screening (Hoppenot et al., 2012). The HPV testing, on the other hand, has higher sensitivity and positive predictive value when compared to cervical cytology (Guo et al., 2007; Mayrand et al., 2007; Hoppenot et al., 2012). Therefore, HPV testing either alone or along with cervical cytology will improve the cervical cancer screening. However, high sensitivity may result in over referral to colposcopy or risk of over treatment (Ronco and Rossi, 2008). For instance, the prevalence of HR-HPV infection in sexually active young women is high and the most infections regress spontaneously in women over 30 years (Fey and Beal, 2004; Rijkaart et al., 2012). Therefore HPV testing is ideally suited for primary screening in women of more than 30 years for better outcome (Sankaranarayanan et al., 2009; Rijkaart et al., 2012; Dijkstra et al., 2014).

HPV testing can be used as a primary screening in a setting where cervical cytology does not exist e.g., developing countries (McAdam et al., 2010). For instance, in a low resource setting where the availability of cytology is limited and burden of cervical cancer is greatest, for women over 30 years, HPV testing could be adopted for effective intervention (McAdam et al., 2010; Schiffman et al., 2011; Ronco et al., 2014; Agorastos et al., 2015). The objective of cervical cancer screening is to detect early phase of precancerous lesions, thereby reducing the incidence and mortality from cervical cancer. And our study, though a hospital-based with limited sample size, indicates that detection of HR- HPV might be a suitable candidate for early identification of cervical precancerous lesions and in the prevention of cervical cancer.

\section{References}

Agorastos T, Chatzistamatiou K, Katsamagkas T, et al (2015). Primary screening for cervical cancer based on high-risk human papillomavirus (HPV) detection and HPV 16 and HPV 18 genotyping, in comparison to cytology. PLoS one, 10, 119755.

Bhatla N, Lal N, BaoYP, Ng T, Qiao YL (2008). A meta-analysis of human papillomavirus type-distribution in women from South Asia: implications for vaccination. Vaccine, 26, 2811-7.

Bosch FX, Burchell AN, Schiffman M, et al (2008). Epidemiology and natural history of human papillomavirus infections and type-specific implications in cervical neoplasia. Vaccine, 26, 1-16.

Bosch FX, Lorincz A, Muñoz N, Meijer CJ, Shah KV (2002). The causal relation between human papillomavirus and cervical cancer. J Clin Pathol, 55, 244-65.

Bosch FX, Qiao YL, Caslellsagué X (2006). Chapter 2: The epidemiology of human papillomavirus infection and its association with cervical cancer. Int J Gynecol Obstet, 94, 8-21.

Burd EM (2003). Human papillomavirus and cervical cancer. 
Krishnan Baskaran et al Clin Microbiol Rew, 16, 1-17.

Clifford G, Franceschi S, Diaz M, Muñoz N, Villa LL (2006). Chapter 3: HPV type-distribution in women with and without cervical neoplastic diseases. Vaccine, 24, 26-34.

Dijkstra MG, Snijders PJF, Arbyn M, et al (2014). Cervical cancer screening: on the way to a shift from cytology to full molecular screening. Ann Oncol, 25, 927-35.

de Villiers EM, Fauquet C, Broker TR, Bernard HU, zur Hausen H (2004). Classification of papillomaviruses. Virology, 324, 17-27.

Duarte-Franco E, Franco EL (2004). Cancer of the uterine cervix. BMC Womens Health, 4, 13.

Fey MC and Beal MW (2004). Role of human papillomavirus testing in cervical cancer prevention. J Midwifery Womens Health, 49, 4-13.

GLOBOCAN 2012. Available at: http://www.iarc.fr

Guo M, Sneige N, Silva EG, et al (2007). Distribution and viral load of eight oncogenic types of human papillomavirus (HPV) and HPV 16 integration status in cervical intraepithelial neoplasia and carcinoma. Mod Pathol, 20, 256-66.

Heymans J, Benoy IH, Poppe W, Depuydt CE (2011). Typespecific HPV geno-typing improves detection of recurrent high-grade cervical neoplasia after conisation. Int J Cancer, 129, 903-9.

Hoppenot C, Stampler K, Dunton C (2012). Cervical cancer screening in high- and low-resource countries: implications and new developments. Obstet Gynecol Surv, 67, 658-67.

Lu KH and Burke TW (2000). Early cervical cancer. Curr Treat Options Oncol, 1, 147-55.

Mayrand MH, Duarte-Franco E, Rodrigues I, et al (2007). Human papillomavirus DNA versus Papanicolaou screening tests for cervical cancer. $N$ Engl J Med, 357, 1579-88.

McAdam M, Sakita J, Tarivonda L, Pang J, Frazer IH (2010). Evaluation of a cervical cancer screening program based on HPV testing and LLETZ excision in a low resource setting. PLoS, 5, 13266.

Moscicki AB, Schiffman M, Kjaer S, Villa LL (2006). Chapter 5: Updating the natural history of HPV and anogenital cancer. Vaccine, 24, 42-51.

Muñoz N, Bosch FX, de Sanjosé S, et al. (2003). Epidemiologic classification of human papillomavirus types associated with cervical cancer. $N$ Engl J Med, 348, 518-27.

Muñoz N, Castellsagué X, de González AB, Gissmann L (2006). Chapter 1: HPV in the etiology of human cancer. Vaccine, 24, 1-10.

Rijkaart DC, Berkhof J, van Kemenade FJ, et al (2012). HPV DNA testing in population-based cervical screening (VUSAScreen study): results and implications. Br J Cancer, 106, 975-81.

Ronco G and Rossi PG (2008). New paradigms in cervical cancer prevention: opportunities and risks. BMC Womens Health, 8, 1-4.

Ronco G, Dillner J, Elfström KM, et al (2014). Efficacy of HPVbased screening for prevention of invasive cervical cancer: follow-up of four European randomised controlled trials. Lancet, 383, 524-32.

Sankaranarayanan R, Nene BM, Shastri SS, et al (2009). HPV screening for cervical cancer in rural India. $N$ Engl J Med, 360, 1385-94.

Schiffman M, Castle PE, Jeronimo J, Rodriguez AC, Wacholder S (2007). Human papillomavirus and cervical cancer. Lancet, 370, 890-907.

Schiffman M, Wentzensen N, Wacholder S, et al (2011). Human papillomavirus testing in the prevention of cervical cancer. J Natl Cancer Inst, 103, 368-83.

Schiffman MH, Bauer HM, Hoover RN, et al (1993). Epidemiologic evidence showing that human papillomavirus infection causes most cervical intraepithelial neoplasia. $J$ Natl Cancer Inst, 85, 958-64.

Smith SJ, Lindsay L, Hoots B, et al (2007). Human papillomavirus type distribution in invasive cervical cancer and high-grade cervical lesions: a meta-analysis update. Int J Cancer, 121, 621-32.

Solomon D, Davey D, Kurman R, et al (2002). The 2001 Bethesda system: terminology for reporting results of cervical cytology. JAMA, 287, 2114-9.

Szostek S, Klimek M, Zawilinska B, Kosz-Vnenchak M (2008). Genotype-specific human papillomavirus detection in cervical smears. Acta Biochim Pol, 55, 687-92.

Tjalma WA, Van Waes TR, Van den Eeden LE, Bogers JJ (2005). Role of human papillomavirus in the carcinogenesis of squamous cell carcinoma and adenocarcinoma of the cervix. Best Pract Res Clin Obstet Gynecol, 19, 469-83.

Villa LL (2006). Chapter 1: Biology of genital human papillomaviruses. Int J Gynecol Obstet, 94, S3-7.

Wright TC Jr (2007). Cervical cancer screening in the 21 st century: is it time to retire the Pap smear? Clin Obstet Gynecol, 50, 313-23. 